\title{
Boron isotopic signatures in cold- water corals determined by femtosecond LA-ICP-MS
}

\author{
GRIT STEINHOEFEL ${ }^{1}$, KRISTINA BECK ${ }^{2}$, MARLENE \\ WALL $^{3}$, GERNOT NEHRKE ${ }^{4}$ AND CHRISTOPH HELD ${ }^{2}$ \\ ${ }^{1}$ Alfred Wegener Institute \\ ${ }^{2}$ Alfred-Wegener Institute \\ ${ }^{3}$ Alfred-Wegener-Institute \\ ${ }^{4}$ Alfred-Wegener Institut: Helmholtz-Zentrum für Polar- und \\ Meeresforschung \\ Presenting Author: grit.steinhoefel@awi.de
}

In this study, we present B isotopic data at high spatial resolution for standard materials and cold-water corals ( $D$. Dianthus) determined by UV femtosecond laser ablation system coupled to a Nu Plasma II MC-ICP-MS.

Multiple analysis of silicate and carbonate standard materials including NIST SRM 612, the MPI-DING series (komatiite to rhyolite glasses), IAEA-B-8 (clay) and JCp-1 (coral) reveal average $\delta^{11} \mathrm{~B}$ values, which agree well with published data. The reproducibility is better than $0.8 \%$ ( $2 \mathrm{SD}$ ). We employed this technique to determine $\mathrm{B}$ isotopic ratios in recent cold-water corals (D. dianthus) from a field experiment in the Comau Fjord (Chile) to resesolve seasonal variations. Corals from the head and the mouth of the fjord and from different water depths were stained with calcein, replaced at the same location or crosstransplanted and observed for a whole year to investigate timeresolved environmental signatures and potential adaptation effects under new environmental conditions. B isotopic ratios were determined at high spatial resolution at the upper part of the calyx in transverse sections between septa at the skeletal increment. $\delta^{11} \mathrm{~B}$ values range between 23.5 and $27.0 \%$ and varies with seasonality and location in the fjord. Isotope signature of cross-transplanted corals do not differ significantly from those of local corals showing that the recorded signature is directly influenced by environmental conditions. B isotopic signatures correspond to an internal $\mathrm{pH}$ up-regulation for calcification of 0.78 to $1.14 \mathrm{pH}$ units, which is likely controlled by ambient seawater $\mathrm{pH}$ and nutrient availability. These results show the potential of in situ techniques to decipher marine archive to reconstruct environmental conditions. 